DOI: $10.15393 / \mathrm{j} 3 . \operatorname{art} .2018 .4690$

UDC 517.444

\author{
V. V. VOLChKOV, Vit. V. VOLChKOV
}

\title{
A NEW CHARACTERIZATION OF HOLOMORPHIC FUNCTIONS IN THE UNIT DISK
}

\begin{abstract}
We study the conditions under which a function satisfying a weighted Morera property for all hyperbolic circles of a fixed radius is holomorphic. We show that one of such conditions is the restriction on a speed of decrease of the difference between the function and its Cauchy type integral.
\end{abstract}

Key words: Cauchy integral formula, holomorphy tests, Legendre functions, hyperbolic plane

2010 Mathematical Subject Classification: $43 A 45$

1. Introduction and the statement of the main result. Let $\mathbb{C}$ be the complex plane, $\mathbb{D}=\{z \in \mathbb{C}:|z|<1\}, \mathbb{T}=\{z \in \mathbb{C}:|z|=1\}$, $\overline{\mathbb{D}}=\mathbb{D} \cup \mathbb{T}$. By the classical Cauchy theorem, the necessary and sufficient condition of a function $f \in C(\overline{\mathbb{D}})$ to be holomorphic in $\mathbb{D}$ is

$$
f(z)=\frac{1}{2 \pi i} \int_{\mathbb{T}} \frac{f(\zeta)}{\zeta-z} d \zeta \quad \text { for all } z \in \mathbb{D} .
$$

Other holomorphy tests which are based on the Cauchi integral formula can be found in [3]. Another characterization of holomorphic functions is related with the well-known Morera property which has been studied in many contexts and generality (we refer the reader to [1], [7]-[9], [11], [12] for an account of considerable amount of research).

Throughout what follows $G$ is the conformal automorphism group of the disk $\mathbb{D}$. We denote by $g A$ the image of a set $A \subset \mathbb{D}$ under the map $g \in G$. For $\varrho \in(0,1)$ we set $\gamma_{\varrho}=\{z \in \mathbb{C}:|z|=\varrho\}$.

Let $f \in C(\mathbb{D})$ and let $E \subset(0,1)$ be a given set. Assume that

$$
\int_{\gamma_{\varrho}} f(g z) d z=0
$$

(C) Petrozavodsk State University, 2018 
for all $g \in G, \varrho \in E$. For which $E$ does this imply that $f$ is holomorphic? One of the results in [2] states that $f$ is holomorphic if and only if the equations

$$
P_{z}^{-1}\left(\frac{1+\varrho^{2}}{1-\varrho^{2}}\right)=0 \quad(\varrho \in E)
$$

have no common solution $z \in \mathbb{C}$. Here and below $P_{z}^{\nu}$ is the associated Legendre function of the first kind (see [4, Ch. 3, Sect. 3.2]). Also, there is a nonconstant radial real analytic (so nonholomorphic) function on $\mathbb{D}$ satisfying (2) for one fixed $\varrho \in(0,1)$ and all $g \in G$.

In this paper we present a new aspect: we study the case when an assumption of type (1) is replaced by an upper bound of the difference

$$
f(z)-\frac{1}{2 \pi i} \int_{\mathbb{T}} \frac{f(\zeta)}{\zeta-z} d \zeta
$$

as $|z| \rightarrow 1$, and assumption (2) holds for one fixed $\varrho \in(0,1)$ and all $g \in G$. Our main result is as follows.

Theorem 1. Let $\varrho \in(0,1)$ be fixed. Then

(i) If $f \in C(\overline{\mathbb{D}})$ satisfies (2) for all $g \in G$ and

$$
f(z)-\frac{1}{2 \pi i} \int_{\mathbb{T}} \frac{f(\zeta)}{\zeta-z} d \zeta=o(\sqrt{1-|z|}) \quad \text { as }|z| \rightarrow 1,
$$

then $f$ is holomorphic on $\mathbb{D}$.

(ii) There exists a nonholomorphic function $f \in C(\overline{\mathbb{D}})$ such that condition (2) is fulfilled for all $g \in G$, and

$$
f(z)-\frac{1}{2 \pi i} \int_{\mathbb{T}} \frac{f(\zeta)}{\zeta-z} d \zeta=O(\sqrt{1-|z|}) \quad \text { as }|z| \rightarrow 1 .
$$

The proof of Theorem 1 is based on the development of the method proposed by the authors in [10]. We introduce a transmutation operator which establishes a homeomorphism between the space of smooth radial functions in $\mathbb{D}$ and the space of even functions in $C^{\infty}(\mathbb{R})$. In a certain general sense it commutes with the generalized convolution operator; this allows us to reduce the problem to the one-dimensional case. Finally, 
we use some results of the theory of convolution equations in $\mathbb{R}$ (see [7, Part 3]).

2. Notation. It is known that for each $g \in G$ there exist uniquely defined parameters $\tau, z \in \mathbb{C}$ such that $|\tau|=1,|z|<1$, and

$$
g w=\tau \frac{w-z}{1-\bar{z} w}
$$

for each $w \in \mathbb{D}$. The group $G$ parametrized by pairs $(\tau, z)$ is the motion group in the Poincaré model of the hyperbolic plane $\mathbb{H}^{2}$ which is realized as the disk $\mathbb{D}$ and has the corresponding Riemannian structure (see [5, Introduction, $\S 4]$ ). The hyperbolic distance $d$ between the points $z_{1}, z_{2} \in$ $\in \mathbb{H}^{2}$ in this model is defined by

$$
d\left(z_{1}, z_{2}\right)=\frac{1}{2} \ln \frac{\left|1-\bar{z}_{1} z_{2}\right|+\left|z_{2}-z_{1}\right|}{\left|1-\bar{z}_{1} z_{2}\right|-\left|z_{2}-z_{1}\right|} .
$$

In particular,

$$
d(z, 0)=\frac{1}{2} \ln \frac{1+|z|}{1-|z|}=\operatorname{arth}|z| \quad \text { and } \quad|z|=\operatorname{th} d(z, 0), \quad z \in \mathbb{H}^{2} .
$$

The hyperbolic measure $d \mu$ on $\mathbb{H}^{2}$ has the form

$$
d \mu(z)=\frac{i}{2} \frac{d z \wedge \overline{d z}}{\left(1-|z|^{2}\right)^{2}}
$$

The distance $d$ and the measure $d \mu$ are invariant with respect to the group $G$.

For $r>0$, the symbol $B_{r}$ denotes the open hyperbolic disk of radius $r$ centered at the origin of $\mathbb{H}^{2}$, i.e.,

$$
B_{r}=\left\{z \in \mathbb{H}^{2}: d(0, z)<r\right\} .
$$

Let $B_{\infty}=\mathbb{H}^{2}$. For $r \geq 0$, we set

$$
\bar{B}_{r}=\left\{z \in \mathbb{H}^{2}: d(0, z) \leq r\right\}, \quad \partial B_{r}=\left\{z \in \mathbb{H}^{2}: d(0, z)=r\right\} .
$$

Furthermore, let $\chi_{r}$ be the characteristic function (the indicator) of the disk $B_{r}$. We denote by $L\left(\mathbb{H}^{2}\right)$ and $L_{\mathrm{loc}}\left(\mathbb{H}^{2}\right)$ the classes of functions integrable and locally integrable on $\mathbb{H}^{2}$ with respect to the measure $d \mu$, 
respectively. Let $d g$ be the Haar measure on $G$ normalized so that the relation

$$
\int_{G} \psi(g 0) d g=\int_{\mathbb{H}^{2}} \psi(z) d \mu(z)
$$

is valid for each function $\psi \in L\left(\mathbb{H}^{2}\right)$ (see [5, Introduction, $\S 4$, Section 3]).

Let $\mathcal{D}\left(\mathbb{H}^{2}\right)$ (or $\mathcal{D}(\mathbb{R})$ ) be the set of functions with compact supports in $C^{\infty}\left(\mathbb{H}^{2}\right)$ (in $C^{\infty}(\mathbb{R})$, respectively) endowed with the standard topology (for instance, see [5, Ch. $2, \S 2.2]$ ). We denote the spaces of radial functions in $L\left(\mathbb{H}^{2}\right), C^{\infty}\left(\mathbb{H}^{2}\right)$ and $\mathcal{D}\left(\mathbb{H}^{2}\right)$ with the induced topology by $L_{\natural}\left(\mathbb{H}^{2}\right)$, $C_{\natural}^{\infty}\left(\mathbb{H}^{2}\right)$ and $\mathcal{D}_{\natural}\left(\mathbb{H}^{2}\right)$. In a similar way, we let $C_{\natural}^{\infty}(\mathbb{R})$ and $\mathcal{D}_{\natural}(\mathbb{R})$ denote the spaces of even functions in $C^{\infty}(\mathbb{R})$ and $\mathcal{D}(\mathbb{R})$, respectively.

Let $f_{1}, f_{2}$ be radial functions in the class $L_{\mathrm{loc}}\left(\mathbb{H}^{2}\right)$. Assume that at least one of the functions $f_{1}$ and $f_{2}$ has compact support. Then we define the generalized hyperbolic convolution $f_{1} \diamond f_{2}$ by

$$
\left(f_{1} \diamond f_{2}\right)(g 0)=\int_{\mathbb{H}^{2}} f_{1}(z) f_{2}\left(g^{-1} z\right) \frac{\left(1-|z|^{2}\right)^{2}}{(1-z \cdot \overline{g 0})^{2}} d \mu(z), \quad g \in G .
$$

Equality (6) shows that $f_{1} \diamond f_{2}$ is a radial function in the class $L_{\mathrm{loc}}\left(\mathbb{H}^{2}\right)$.

It follows from (6) and (5) that

$$
\left(f_{1} \diamond f_{2}\right)(\zeta)=\int_{G} f_{1}(g 0) f_{2}\left(g^{-1} \zeta\right) \frac{\left(1-|g 0|^{2}\right)^{2}}{(1-\bar{\zeta} \cdot g 0)^{2}} d g, \quad \zeta \in \mathbb{D},
$$

and

$$
f_{1} \diamond f_{2}=f_{2} \diamond f_{1} .
$$

If $f_{1}, f_{2} \in C_{\natural}^{\infty}\left(\mathbb{H}^{2}\right)$ then, in view of (7),

$$
\mathfrak{L}\left(f_{1} \diamond f_{2}\right)=f_{1} \diamond \mathfrak{L} f_{2}=\left(\mathfrak{L} f_{1}\right) \diamond f_{2}
$$

where

$$
\mathfrak{L}=4\left(1-|z|^{2}\right)^{2} \frac{\partial^{2}}{\partial z \partial \bar{z}}-8\left(1-|z|^{2}\right) z \frac{\partial}{\partial z} .
$$

In addition, if $f_{1}, f_{2}, f_{3} \in L_{\mathrm{loc}}\left(\mathbb{H}^{2}\right)$ are radial functions, and at least two of the functions $f_{1}, f_{2}, f_{3}$ have compact supports, then

$$
\left(f_{1} \diamond f_{2}\right) \diamond f_{3}=f_{1} \diamond\left(f_{2} \diamond f_{3}\right) .
$$


Let $h \in L(\mathbb{R})$. Its Fourier transform $\widehat{h}$ is defined by

$$
\widehat{h}(\lambda)=\int_{-\infty}^{+\infty} h(t) e^{-i \lambda t} d t, \quad \lambda \in \mathbb{R} .
$$

Assume that $\lambda \in \mathbb{C}$ and

$$
\nu=\nu(\lambda)=\frac{i \lambda-1}{2} .
$$

Now define

$$
U_{\lambda}(z)=\left(1-|z|^{2}\right)^{\nu+1} F\left(\nu+2, \nu+1 ; 2 ;|z|^{2}\right), \quad z \in \mathbb{D},
$$

where $F$ is the Gauss hyperheometric function.

Also let

$$
\mathcal{H}_{\lambda}(z)=\left(1-|z|^{2}\right)^{\nu} F\left(\nu+2, \nu ; 1 ;|z|^{2}\right), \quad \lambda \in \mathbb{C}, z \in \mathbb{D} .
$$

We can conclude from (11) and the expansion of $F$ in a hypergeometric series that $\mathcal{H}_{\lambda}$ is an entire function of $\lambda$. It is even because

$$
\mathcal{H}_{\lambda}(z)=\left(1-|z|^{2}\right)^{-2} F\left(\frac{3+i \lambda}{2}, \frac{3-i \lambda}{2} ; 1 ; \frac{|z|^{2}}{|z|^{2}-1}\right)
$$

(see [4, formula $2.9(3)])$ and $F(a, b ; c ; z)$ is symmetric in $a$ and $b$. It follows from $(9), 11)$ and the hypergeometric differential equation that

$$
\left(\mathfrak{L} \mathcal{H}_{\lambda}\right)(z)=-\left(\lambda^{2}+1\right) \mathcal{H}_{\lambda}(z) .
$$

Suppose that the function $T \in L_{\natural}\left(\mathbb{H}^{2}\right)$ is compactly supported. For $\lambda \in \mathbb{C}$, let

$$
\mathcal{F}(T)(\lambda)=\int_{\mathbb{H}^{2}} T(z) \mathcal{H}_{\lambda}(z)\left(1-|z|^{2}\right)^{2} d \mu(z) .
$$

Equality 12 shows that the function $\mathcal{F}(T)$ is an even entire function of $\lambda$. If $f_{1}$ and $f_{2}$ are functions with compact support in $L_{\natural}\left(\mathbb{H}^{2}\right)$ then

$$
\mathcal{F}\left(f_{1} \diamond f_{2}\right)=\mathcal{F}\left(f_{1}\right) \mathcal{F}\left(f_{2}\right)
$$

(see (8), 13) and the proof of Lemma 8 in [10]). 
3. Auxiliary results. We need the following lemmas in the proof of Theorem 1 .

Lemma 1. The following equality holds

$$
\frac{\partial}{\partial \bar{z}}\left(\bar{z} U_{\lambda}(z)\right)=2 \mathcal{H}_{\lambda}(z), \quad \lambda \in \mathbb{C}, z \in \mathbb{D},
$$

where the function $U_{\lambda}$ is defined by 10 .

Proof. First assume that $z \neq 0$. Relation 10 yields

$$
\frac{\partial}{\partial \bar{z}}\left(\bar{z} U_{\lambda}(z)\right)=\frac{1}{\rho} \frac{d}{d \rho}\left(\rho^{2}\left(1-\rho^{2}\right)^{\nu+1} F\left(\nu+2, \nu+1 ; 2 ; \rho^{2}\right)\right) .
$$

Using now $[4$, formula $2.8(25)]$ and $(11)$ we arrive at $(15)$. Now continuous extension to the point $z=0$ completes the proof.

For $\alpha, \beta, \lambda \in \mathbb{C}$, we set

$$
\varphi_{\lambda}^{(\alpha, \beta)}(r)=F\left(\frac{\alpha+\beta+1-i \lambda}{2}, \frac{\alpha+\beta+1+i \lambda}{2} ; \alpha+1 ;-\operatorname{sh}^{2} r\right) .
$$

Lemma 2. For each $\lambda \in \mathbb{C}$,

$$
\mathcal{F}\left(\chi_{r}\right)(\lambda)=\pi \operatorname{sh}^{2} r \varphi_{\lambda}^{(1,1)}(r) .
$$

Proof. For brevity we set

$$
z=-\operatorname{sh}^{2} r, \quad a=\frac{1+i \lambda}{2}, \quad b=\frac{1-i \lambda}{2} .
$$

The expansion of $\varphi_{\lambda}^{(\alpha, \beta)}(r)$ in a hypergeometric series shows that

$$
\begin{gathered}
\lim _{\alpha \rightarrow-1} \frac{\varphi_{\lambda}^{(\alpha, 1)}(r)-1}{\Gamma(\alpha+1)}=\sum_{n=1}^{\infty} \frac{a(a+1) \ldots(a+n-1) b(b+1) \ldots(b+n-1)}{n ! \Gamma(n)} z^{n}= \\
=z \sum_{n=0}^{\infty} \frac{a(a+1) \ldots(a+n) b(b+1) \ldots(b+n)}{n ! \Gamma(n+2)} z^{n}=a b z F(a+1, b+1 ; 2 ; z)= \\
=-\frac{1}{4}\left(\lambda^{2}+1\right) \operatorname{sh}^{2} r \varphi_{\lambda}^{(1,1)}(r) .
\end{gathered}
$$


Now, by the definition of the transform $\mathcal{F}$ and [8, Proposition 7.2 (ii)] we see that

$$
\mathcal{F}\left(\chi_{r}\right)(\lambda)=\pi \int_{0}^{r} \operatorname{sh}(2 t) \varphi_{\lambda}^{(0,2)}(t) d t=-\frac{4 \pi}{\lambda^{2}+1} \lim _{\alpha \rightarrow-1} \frac{\varphi_{\lambda}^{(\alpha, 1)}(r)-1}{\Gamma(\alpha+1)} .
$$

This gives, by the equality above, the desired result.

Lemma 3. For each $r>0$, the following assertions hold.

(i) $\mathcal{F}\left(\chi_{r}\right)(0) \neq 0$.

(ii) The function $\mathcal{F}\left(\chi_{r}\right)(\lambda)$ has infinitely many zeros, all of which are real, simple and lie symmetrically relative to $\lambda=0$.

(iii) If $\mathcal{F}\left(\chi_{r}\right)(\lambda)=0$ then $\left|\mathcal{F}\left(\chi_{r}\right)^{\prime}(\lambda)\right|>c|\lambda|^{-3 / 2}$, where the constant $c>0$ is independent of $\lambda$.

Proof. Using (16) and [4, formula 3.2(7)] we obtain

$$
\mathcal{F}\left(\chi_{r}\right)=\pi \operatorname{th} r P_{(i \lambda-1) / 2}^{-1}(\operatorname{ch} 2 r) .
$$

Now the assertions of Lemma 3 are partial cases of Lemmas 2.4, 2.5 and Corollary 2.2 in [7, Part 2, Ch. 2].

The next statements are analogs of the Paley-Wiener theorem and the inversion formula for the transform $\mathcal{F}$.

\section{Lemma 4.}

(i) An even entire function $w$ is the $\mathcal{F}$-transform of a function in $\mathcal{D}_{\natural}\left(\mathbb{H}^{2}\right)$ with support in $\bar{B}_{r}$ if and only if for each $N \in \mathbb{Z}_{+}$there exists a positive constant $c_{N}>0$ such that

$$
|w(\lambda)| \leq c_{N} \frac{e^{r|\operatorname{Im} \lambda|}}{(1+|\lambda|)^{N}}, \quad \lambda \in \mathbb{C} .
$$

(ii) Assume that $f \in\left(L_{\natural} \cap C\right)\left(\mathbb{H}^{2}\right)$ and

$$
\int_{0}^{\infty} \lambda|\mathcal{F}(f)(\lambda)| d \lambda<+\infty
$$

Then

$$
f(z)=\frac{16}{\pi^{2}} \int_{0}^{\infty} \mathcal{F}(f)(\lambda) \mathcal{H}_{\lambda}(z)|c(\lambda)|^{-2} d \lambda+\frac{1}{2 \pi} \mathcal{F}(f)(i) \mathcal{H}_{i}(z),
$$


where

$$
c(\lambda)=\frac{2^{3-i \lambda} \Gamma(i \lambda)}{\Gamma\left(\frac{i \lambda-1}{2}\right) \Gamma\left(\frac{i \lambda+3}{2}\right)}
$$

and the integral is absolutely convergent for each $z \in \mathbb{C}$.

Proof. To prove (i), let $0<\xi<t$. We set

$$
K(t, \xi)=\frac{2^{3 / 2}}{\pi}(\operatorname{ch} t)^{2}(\operatorname{ch} 2 t-\operatorname{ch} 2 \xi)^{-1 / 2} F\left(2,-2 ; \frac{1}{2} ; \frac{\operatorname{ch} t-\operatorname{ch} \xi}{2 \operatorname{ch} t}\right) .
$$

For $\lambda \in \mathbb{C}, z \in \mathbb{D}$, we have

$$
\mathcal{H}_{\lambda}(z)=\int_{0}^{t} K(t, \xi) \cos \lambda \xi d \xi
$$

where $t=\operatorname{arth}|z|$ (see [8, Proposition 7.3]). Owing to (13), the rest of the proof is identical to that of Proposition 1 in [10].

In view of Theorem 2.3 in [6], assertion (ii) can be proved in the same way as Proposition 2 in [10].

For $f \in \mathcal{D}_{\natural}\left(\mathbb{H}^{2}\right), t \in \mathbb{R}^{1}$, let

$$
\mathfrak{A}(f)(t)=\frac{16}{\pi^{2}} \int_{0}^{\infty} \mathcal{F}(f)(\lambda)|c(\lambda)|^{-2} \cos (\lambda t) d \lambda+\frac{1}{2 \pi} \mathcal{F}(f)(i) \cos (i t) .
$$

Using Lemma 4 and Stirling's formula, it is easy to see that $\mathfrak{A}(f) \in$ $\in C_{\natural}^{\infty}\left(\mathbb{R}^{1}\right)$.

Suppose that $T \in L_{\natural}\left(\mathbb{H}^{2}\right)$ has compact support. If $\mathcal{F}(T) \in L^{2}\left(\mathbb{R}^{1}\right)$ then, by the classical Paley-Wiener theorem, there exists a function $\Lambda(T) \in$ $\in L^{2}\left(\mathbb{R}^{1}\right)$ with compact support such that $\widehat{\Lambda(T)}=\mathcal{F}(T)$.

The main properties of the map $f \rightarrow \mathfrak{A}(f)$ are contained in the following lemma.

\section{Lemma 5.}

(i) The transformation $\mathfrak{A}$ can be extended to a linear homeomorphism of the spaces $C_{\natural}^{\infty}\left(\mathbb{H}^{2}\right)$ and $C_{\natural}^{\infty}\left(\mathbb{R}^{1}\right)$.

(ii) Let $f \in C_{\natural}^{\infty}\left(\mathbb{H}^{2}\right), r>0$. Then $f=0$ in $B_{r}$ if and only if $\mathfrak{A}(f)=0$ in $(-r, r)$. 
(iii) Assume that $T \in L_{\natural}\left(\mathbb{H}^{2}\right)$ has compact support and let $\mathcal{F}(T) \in$ $\in L^{2}\left(\mathbb{R}^{1}\right)$. Then

$$
\mathfrak{A}(f \diamond T)(t)=\mathfrak{A}(f) * \Lambda(T)
$$

for each $f \in C_{\natural}^{\infty}\left(\mathbb{H}^{2}\right)$.

(iv) Let $\lambda \in \mathbb{C}$. Then

$$
\mathfrak{A}\left(\mathcal{H}_{\lambda}\right)(t)=\cos \lambda t
$$

Proof. This lemma can be proved in the same way as Theorems 2 and 3 in [10] taking into account Lemma 4 and (14).

4. Proof of the main result. We now proceed to the proof of Theorem 1. To prove (i), we remark that we may confine our attention to the case $f \in C(\overline{\mathbb{D}}) \cap C^{\infty}(\mathbb{D})$ (otherwise we can use the standard hyperbolic regularization, see [5, Ch. 1, the proof of Theorem 4.2]). Let $r>0$ and

$$
J_{f}(\zeta)=\frac{1}{1-|\zeta|^{2}} \int_{\partial B_{r}} f\left(\frac{z+\zeta}{1+\bar{\zeta} z}\right) d z, \quad \zeta \in \mathbb{D}
$$

We set

$$
g_{\zeta} z=\frac{z+\zeta}{1+\bar{\zeta} z}, \quad z \in \mathbb{D}
$$

By Green's formula, we have

$$
J_{f}(\zeta)=-\int_{B_{r}} \frac{\partial}{\partial \bar{z}}\left(f\left(g_{\zeta} z\right)\right) \frac{d z \wedge d \bar{z}}{1-|\zeta|^{2}}=2 i \int_{B_{r}} \frac{\partial f}{\partial \bar{z}}\left(g_{\zeta} z\right) \frac{\left(1-|z|^{2}\right)^{2}}{(1+\zeta \bar{z})^{2}} d \mu(z)
$$

Since $g_{\zeta} \in G$ and the measure $d \mu(z)$ is $G$-invariant,

$$
J_{f}(\zeta)=2 i \int_{g_{\zeta} B_{r}} \frac{\partial f}{\partial \bar{z}}(w) \frac{\left(1-\left|g_{\zeta}^{-1} w\right|^{2}\right)^{2}}{\left(1+\zeta \overline{g_{\zeta}^{-1} w}\right)^{2}} d \mu(w)
$$

This implies easily that

$$
J_{f}(\zeta)=2 i \int_{g_{\zeta} B_{r}} \frac{\partial f}{\partial \bar{z}}(w) \frac{\left(1-|w|^{2}\right)^{2}}{(1-w \bar{\zeta})^{2}} d \mu(w) .
$$


Because of (6) we can write

$$
J_{f}=2 i \frac{\partial f}{\partial \bar{z}} \diamond \chi_{r} \quad \text { in } \quad \mathbb{H}^{2} .
$$

Next, let $g \in G, z \in \mathbb{D}$, and

$$
g z=\frac{a z+b}{\bar{b} z+\bar{a}}, \quad \text { where } \quad a, b \in \mathbb{C}, \quad|a|^{2}-|b|^{2}=1 .
$$

Putting $\zeta=g 0$, we see from 19 and 31 that

$$
J_{f}(g 0)=|a|^{2} \int_{\partial B_{r}} f\left(\frac{z+\frac{b}{\bar{a}}}{1+\frac{\bar{b}}{a} z}\right) d z .
$$

The change of variable $z=\frac{a}{\bar{a}} w$ in the integral enables us to write

$$
J_{f}(g 0)=a^{2} \int_{\partial B_{r}} f(g z) d z \quad \forall g \in G
$$

Equalities (20) and (22) show that the function $f$ satisfies (2) for all $g \in G$ if and only if

$$
\frac{\partial f}{\partial \bar{z}} \diamond \chi_{r}=0 \quad \text { in } \mathbb{H}^{2}
$$

where $r=\operatorname{arth} \rho$. Introduce the following auxiliary function

$$
u(z)=\frac{1}{2 \pi i} \int_{|\zeta|=1} f(\zeta z) d \zeta, \quad z \in \mathbb{D} .
$$

It follows from $(24)$ that $u \in C^{\infty}(\mathbb{D})$. We then find that $u$ satisfies $(2)$ and, if $z \neq 0$,

$$
u(z)=v(|z|) e^{-i \arg z}, \quad \text { where } \quad v(|z|)=\frac{1}{2 \pi} \int_{-\pi}^{\pi} f\left(|z| e^{i \varphi}\right) e^{i \varphi} d \varphi .
$$

A straightforward calculation shows that

$$
\frac{\partial u}{\partial \bar{z}}=v^{\prime}(|z|)+\frac{v(|z|)}{|z|} .
$$


In particular, $\frac{\partial u}{\partial \bar{z}} \in C_{\natural}^{\infty}\left(\mathbb{H}^{2}\right)$. In addition, it follows from 25 that

$$
v(0)=0
$$

Relation (23) leads to

$$
\frac{\partial u}{\partial \bar{z}} \diamond \chi_{r}=0 \quad \text { in } \quad \mathbb{H}^{2}, \quad \text { where } \quad r=\operatorname{arth} \rho .
$$

Then we find from $(16)$ and the asymptotic expansion for $\varphi_{\lambda}^{(1,1)}(r)$ (see [7, Part 2, Corollary 2.2]) that $\mathcal{F}\left(\chi_{r}\right) \in L^{2}\left(\mathbb{R}^{1}\right)$. By the Paley-Wiener theorem, there exists a function $\Lambda_{r} \in L^{2}\left(\mathbb{R}^{1}\right)$ with compact support such that $\widehat{\Lambda}_{r}=\mathcal{F}\left(\chi_{r}\right)$. Owing to Lemma 5 and (17),

$$
\mathfrak{A}\left(\frac{\partial u}{\partial \bar{z}}\right) * \Lambda_{r}=0 \quad \text { on } \quad \mathbb{R} .
$$

Using now Lemma 3 and [7, Part 3, Theorem 1.3], we conclude that

$$
\mathfrak{A}\left(\frac{\partial u}{\partial \bar{z}}\right)(t)=\sum_{\lambda \in N(r)} c_{\lambda} \cos (\lambda t), \quad t \in \mathbb{R},
$$

where $N(r)=\left\{\lambda>0: \mathcal{F}\left(\chi_{r}\right)=0\right\}, c_{\lambda} \in \mathbb{C}$, the series converges in the space $C^{\infty}(\mathbb{R})$, and

$$
\left|c_{\lambda}\right|=O\left((1+\lambda)^{-\alpha}\right) \quad \text { as } \quad \lambda \rightarrow+\infty
$$

for each fixed $\alpha>0$. According to Lemma 5 the series

$$
\sum_{\lambda \in N(r)} c_{\lambda} \mathcal{H}_{\lambda}
$$

converges in $C^{\infty}\left(\mathbb{H}^{2}\right)$ to some function $w \in C_{\natural}^{\infty}\left(\mathbb{H}^{2}\right)$. Relations 29) and (18) yield

$$
\mathfrak{A}\left(\frac{\partial u}{\partial \bar{z}}\right)=\mathfrak{A}(w)
$$

whence $\frac{\partial u}{\partial \bar{z}}=w$ in $\mathbb{D}$. By the definition of $w$ this shows that

$$
v(|z|)=\frac{|z|}{2} \sum_{\lambda \in N(r)} c_{\lambda} U_{\lambda}(|z|)
$$


(see (26), 27) and Lemma 1). Next, it follows from (25) and (3) that

$$
v(|z|)=o(\sqrt{1-|z|}) \quad \text { as } \quad|z| \rightarrow 1 .
$$

In addition, for each $\varepsilon>0, \lambda \in N(r)$ we have

$$
U_{\lambda}(z)=\left(a(\lambda) e^{i \lambda t}+a(-\lambda) e^{-i \lambda t}\right) e^{-t}+O\left(e^{-2 t}\right),
$$

where

$$
a(\lambda)=\frac{\Gamma(i \lambda / 2)}{\sqrt{\pi} \Gamma((i \lambda+3) / 2)},
$$

$|z|=$ th $\mathrm{t}>\varepsilon$ and the constant in the symbol $O$ depends only on $\varepsilon$ (see [5, Introduction, Theorem 4.15]). Applying now (31) we see from (30) and 32 that

$$
\sum_{\lambda \in N(r)} c_{\lambda}\left(a(\lambda) e^{i \lambda t}+a(-\lambda) e^{-i \lambda t}\right) \rightarrow 0 \quad \text { as } \quad t \rightarrow+\infty .
$$

Together with (33) this implies that $c_{\lambda}=0$ for all $\lambda \in N(r)$ (see, for instance, [7, Part 3, Theorem 1.6]). Owing to (30) we obtain $v=0$.

In view of (25) and (24) this means that

$$
\int_{\gamma_{R}} f(z) d z=0
$$

for each $R \in(0,1)$.

Assume now that $h \in G$. Writing (2) with $f(h z)$ instead of $f$ and using (3) with $h z$ instead of $z$, we obtain

$$
\int_{\gamma_{R}} f(h z) d z=0
$$

for all $R \in(0,1)$. Then

$$
\frac{\partial f}{\partial \bar{z}} \diamond \chi_{r}=0 \quad \text { in } \quad \mathbb{H}^{2} \quad \text { for each } \quad r>0 .
$$

Now, from the arbitrariness of $r>0$ it follows that $f$ is holomorphic.

To prove (ii) consider the function $f(z)=\bar{z} U_{\lambda}(z)$, where $\lambda \in N(r)$ and $r=\operatorname{arth} \rho$. Owing to Lemma 1, we infer that 


$$
\frac{\partial f}{\partial \bar{z}}=2 \mathcal{H}_{\lambda},
$$

whence $f$ is nonholomorphic in $\mathbb{D}$. Next, the proof of (i) shows that $f$ satisfies (28) and (2) for all $g \in G$. Finally, it follows from [5, Introduction, Theorem 4.15] that relation (4) is fulfilled. This completes the proof of Theorem 1.

\section{References}

[1] Berenstein C. A., Chang D. C., Pascuas D., Zalcman L. Variations on the theorem of Morera. Contemp. Math., 1992, vol. 137, pp. 63-78.

[2] Berenstein C. A., Pascuas D. Morera and mean-value type theorems in the hyperbolic disk. Israel J. Math., 1994, vol. 86, pp. 61-106.

[3] Cascante C., Pascuas D. Holomorphy tests based on Cauchy's integral formula. Pacific J. Math., 1995, vol. 171, no. 1, pp. 89-116.

[4] Erdélyi A., Magnus W., Oberhettinger F., Tricomi F. G. Higher transcendental functions. New-York: McGraw-Hill, 1953, vols. 1, 2.

[5] Helgason S. Groups and geometric analysis. Pure Appl. Math., vol. 113, Orlando, FL: Academic Press, 1984.

[6] Koornwinder T. H. Jacobi functions and analysis on noncompact semisimple Lie groups. Special Functions: Group Theoretical Aspects and Applications (R.A. Askey et al. (eds.)). Dordrecht: D. Reidel Publishing Company, 1984, pp. 1-85.

[7] Volchkov V. V. Integral Geometry and Convolution Equations. Dordrecht: Kluwer Academic Publishers, 2003, 454 p.

[8] Volchkov V. V., Volchkov Vit. V. Harmonic Analysis of Mean Periodic Functions on Symmetric Spaces and the Heisenberg Group. London: Springer-Verlag, 2009, 671 p.

[9] Volchkov V. V., Volchkov Vit. V. Offbeat Integral Geometry on Symmetric Spaces. Basel: Birkhäuser, 2013, 592 p.

[10] Volchkov V. V., Volchkov Vit. V. Spectral analysis on the group of conformal automorphisms of the unit disc. Sb.: Math., 2016, vol. 207, no. 7, pp. 942-969.

[11] Zalcman L. A bibliographic survey of the Pompeiu problem. Approximation by solutions of partial differential equations (ed. Fuglede B. et. al). Dordrecht: Kluwer, 1992, pp. 185-194.

[12] Zalcman L. Supplementary bibliography to "A bibliographic survey of the Pompeiu problem". Contemp. Math. Radon Transform and Tomography, 2001, vol. 278, pp. 69-74. 
Received December 22, $201 \%$.

In revised form, March 11, 2018.

Accepted March 11, 2018.

Published online April 24, 2018.

Valerii V. Volchkov

Donetsk National University

24, Universitetskaya str., Donetsk 83001

E-mail: valeriyvolchkov@gmail.com

Vitalii V. Volchkov

Donetsk National University

24, Universitetskaya str., Donetsk 83001

E-mail: volna936@gmail.com 